\title{
Kappa-PSO-FAN based method for damage identification on composite structural health monitoring
}

\author{
Mario A. de Oliveira ${ }^{\mathrm{a}, *}$, Nelcileno V.S. Araujo ${ }^{\mathrm{b}}$, Daniel J. Inman ${ }^{\mathrm{c}}$, Jozue Vieira Filho ${ }^{\mathrm{d}}$ \\ a IFMT- Federal Institute of Education, Science and Technology of Mato Grosso, Department of Electrical and Electronic, Campus Cuiabá, Cuiabá, Mato \\ Grosso, Brazil \\ b UFMT- Federal University of Mato Grosso, Institute of Computing, Cuiabá, Brazil \\ ' University of Michigan, Department of Aerospace Engineering, Ann Arbor, MI, USA \\ d UNESP - Universidade Estadual Paulista, Department of Telecommunication Engineering, Campus de São João da Boa Vista, São Paulo, Brazil
}

\section{A R T I C L E I N F O}

\section{Article history:}

Received 3 April 2017

Revised 2 October 2017

Accepted 10 November 2017

Available online 11 November 2017

\section{Keywords:}

SHM

Electromechanical Impedance

PZT

Intelligent systems

Particle Swarm Optimization

Neural network

\begin{abstract}
A B S T R A C T
Recently much research has been conducted towards finding fast and accurate pattern classifiers applied to Structural Health Monitoring (SHM) systems. In this way, researchers have proposed new methods based on Fuzzy ARTMAP Network (FAN) in order to enhance the success rate for structural damage classification applied to SHM applications. Conversely, the performance of methods based on FAN is very dependent of its setup parameters. In several SHM approaches in the literature, authors have proposed selecting those parameters by using several attempts (empirical and manual selection) and keeping them fixed for all cases in the resulting analysis, hampering the success rate of the neural network. To overcoming that, this paper introduces a new strategy for enhancement of structural damage identification focusing on supervised learning of FAN by using Particle Swarm Optimization (PSO) for selecting optimal setup parameters automatically for the FAN algorithm. Also, the Kappa coefficient is used as an objective function to be maximized through the PSO algorithm. As a result, the optimum setup parameters improved the success rate while the damage identification is being carried out. Indeed this proposed method is certainly very promising and constitutes a novelty. The proposed method achieves more than $75 \%$ hit rate that is significantly higher than the state-of-the-art approaches as presented in this paper. Furthermore, this approach yields a $20 \%$ improvement when considering the worst case scenario. Hence, this approach shows a practical application of expert and intelligent systems applied to damage identification in SHM systems. To conclude, the proposed approach successfully identifies structural damage with accuracy and efficiency.
\end{abstract}

(c) 2017 Elsevier Ltd. All rights reserved.

\section{Introduction}

Global aviation has increased mainly due to the large fleet growth prospects for the next years. According to Lewis (2012), the capacity for aircraft delivery has been significantly increased since the $90 \mathrm{~s}$. For example, in 1980 the ability was 30\%, rising to $36 \%$ and $50 \%$ in 1990 and 2000, respectively. In 2010 the number rose to $60 \%$ and the forecast for 2031 is hitting $65 \%$. Likewise, the use of advanced composite materials in aeronautical structures has followed the same trend of fleet growth. Unlike metals, composites are made up from combinations of different materials both in their composition and shape. Accordingly, the constituent materi-

\footnotetext{
* Corresponding author.

E-mail addresses: mario.oliveira@cba.ifmt.edu.br (M.A. de Oliveira), nelcileno@ic.ufmt.br (N.V.S. Araujo), daninman@umich.edu (D.J. Inman), jozue.vieira@sjbv.unesp.br (J.V. Filho).
}

als retain their identities in the compounds and do not dissolve or merge completely into one another, although they act in unison. Moreover, these materials are much more complex when compared to metallic materials due to their anisotropic characteristics.

Nevertheless, composite structures are also susceptible to structural failure. The most commons types of damage in composite structures, which are in operation, consist of: environmental damage, impact, fatigue, local cracking, debonding, delamination, fracture and erosion of the fiber (Duan \& Ye, 2002). Therefore, Structural Health Monitoring (SHM) methods have been widely investigated over the last few decades because they allow us to evaluate various types of structures under different conditions. Evaluation of such structures must be carried out after having been used for a certain period of time in order to prevent failures. Hence, within the whole context of structures, one of the most important and promising applications is structural evaluation of aircraft. For example, a survey was conducted which shows that nearly $61 \%$ 
of structural analysis in aircraft is carried out by visual inspection. Around 31\% uses another form of Non-Destructive Evaluation (NDE) methods and additionally the inspection is carried out in an unplanned manner. Only 8\% use NDE techniques with a planned maintenance schedule (Brand \& Boller, 1999).

SHM methods based on the Electromechanical Impedance (EMI) have been applied and found useful for evaluating damage in composite structures. Methods based on the EMI principle, as applied to SHM, were initially proposed by Liang, Sun, and Rogers (2014) and certainly the method constitutes an important aspect of Non-Destructive Evaluation (NDE). Currently, several papers based on EMI have been produced. Actually there is a vast literature about the EMI features and its applications (Baptista \& Vieira Filho, 2009; Liang et al., 2014; Park \& Inman, 2007; Park, Cudney, \& Inman, 2000; Park, Sohn, Farrar, \& Inman, 2003). This technique requires that the structure is excited in a frequency range through appropriate transducers PZT (Lead Zirconate Titanate) glued to the structure under evaluation. The monitored structure, including the PZT, is represented by an electromechanical model of mass-spring with a single degree of freedom (Liang et al., 2014). Such method uses PZT transducers glued onto the monitored structure and requires a voltage excitation of low amplitude (typically less than $3 \mathrm{~V}$ ) to produce a forced excitation of the structure. Generally, in this technique, the excitation signal scans over a range of variable frequency from zero up to thousands of $\mathrm{Hz}$ generating small wavelengths for the excitation signal, which contribute significantly to the ability in detecting small damage states often missed by low frequency methods (Baptista \& Vieira Filho, 2009; De Oliveira, Vieira Filho, Lopes, \& Inman, 2016; Park \& Inman, 2007). In practice, a variable frequency source makes the structure vibrate at its natural frequencies and these responses are used to estimate the Frequency Response Function (FRF) and then compute EMI. Indeed, the presence of damage will change these natural frequencies causing shifts in frequency and amplitude. Nonetheless, when applied to composite materials these methods are challenging due to increased damping in the impedance signatures (Na \& Lee, 2012) provided by composites. PZT patches cover a small sensing area, around $0.4 \mathrm{~m}$ (radius), when applied to aluminum structures (Park et al., 2003). Yet the coverage area of the PZT may be drastically reduced for a composite structure by using higher frequencies (EMI-based methods), constituting in a difficult problem, one of the most pressing in the field of SHM (Na \& Lee, 2012; Park \& Inman, 2007).

Previously, several different approaches based on Artificial Neural Networks (ANN) in the context of SHM applications have been addressed in literature (Lopes Jr, Park, Cudney, \& Inman, 2000; Papatheou, Dervilis, Maguire, Antoniadou, \& Worden, 2015; Saxena \& Saad, 2007). Recently, new classes of artificial networks such as the Probabilistic Neural Network (PNN) and the Fuzzy ARTMAP Network (FAN) have shown very promising results for structural damage identification. One reason for the increasing efforts in the related research is that those methods can be applied to different types of structures and several different damage scenarios. For example, methods based on PNN, applied to damage identification in SHM, are addressed in Na and Lee (2013), Palomino, Steffen, and Finzi Neto (2014) and Selva, Cherrier, Bundinger, Lachaud, and Morlierb (2013). According to Na and Lee (2013), methods based on PNN present a faster training procedure and they are easier to implement than the traditional Back-Propagation based methods. More recently, methods based on FAN applied to SHM have been proposed in the literature as follows. Firstly, FAN methods along with wavelets were proposed in Lima et al. (2014a, b). Therein, the results hit a success rate of $100 \%$ for failure identification. Unfortunately, the authors used only simulated scenarios in the implementation. Ali, Saidi, Mouelhi, Morello, and Fnaiech (2015) pinpoint that the Simplified Fuzzy ARTMAP Network (SFAN) presents sev- eral advantages due to being superior suited for either implementation in large-scale systems or on-line based methods and also presents the best performance in terms of training/testing speed when compared to the Back-Propagation network. Subsequently, De Oliveira and Inman (2016) propose a method based on SFAN applied to structural damage assessment in composite structures by using the EMI. The method is promising but it requires expert intervention for the analyses of the best setting parameters for SFAN. De Oliveira and Inman (2017) present a comparative analysis of SFAN and PNN algorithms in the context of identifying structural damage growth. In that paper the authors addressed an analysis in terms of the influence of the SFAN setup parameters by varying those parameters through different values. They point out that SFAN setup parameters have a substantial influence on the SFAN performance. It is important to mention that the authors carried out the analysis based on experimental tests (several attempts) to choose the best setup parameters. In essence, the authors conclude that although SFAN is much more suited then PNN in the context of damage growth. Also, the choice of SFAN setup parameters requires substantial effort and time. To be complete, in many approaches shared in literature (Ali et al., 2015; De Oliveira \& Inman, 2016, 2017; Lima et al., 2014a, b) the authors have fixed the setup parameters constituting a drawback because each application problem/dataset has different features demanding variable setup parameters in order to improving the success rate during damage identification.

In order to enhance the success rate, FAN/SFAN methods require an expert to choose the best setup parameters (choice parameter $\alpha$, training rate $\beta$, network vigilance parameter $\rho$ and match tracking $\varepsilon$ ) for both training and test phases. According to Granger, Renniges, Sabourin, and Oliveira (2007) FAN performance depends on a set of user-defined hyper-parameters which should be carefully tuned to each specific problem. The authors also point out that there are other different solutions, however they focused on exploring the influence of the vigilance parameter, leaving aside others (choice parameter, training rate and match tracking). They also mention that the wrong choice of setup parameters may lead to overtraining and degrading the capacity of generalization of the network. As a solution, they propose using the PSO algorithm to optimize FAN hyper-parameters values. Garcia-Breijo, Garrigues, Sanchez, and Laguarda-Miro (2013) address an embedded SFAN method which was implemented on a microcontroller for food classification. They also point out that the problem of choosing the best SFAN parameters setup. They proposed using different training rates $(\beta)$ and vigilance parameters $(\rho)$ values in order to search for the best recognition rates to select the smallest weight matrix and map field sizes. Unfortunately, they did not mention how to choose the best choice parameter $(\alpha)$ and match tracking $(\varepsilon)$. De Oliveira and Inman $(2016,2017)$ mention that SFAN parameters were chosen based on several attempts (practical tests) which demands a long time and constitutes difficulty with the method. As observed from the literature, there is no consensus about the best way to choose the FAN/SFAN setup parameters and certainly it still constitutes in an open field for more investigation.

From a critical review of the research literature, optimum setup parameters must improve the success rate while the damage identification is being carried out. Hence, a method which allows choosing those optimal parameters automatically is certainly very promising and also constitutes a novelty. A promising and forthcoming way to obtain optimal FAN parameters consists of using the Particle Swarm Optimization (PSO) algorithm (Granger et al., 2007). Unfortunately, PSO based methods have not been applied to SHM approaches until recently. Only a few efforts have focused on SHM-PSO based methods. This previous research is summarized in the following. One of the earliest works on SHM-PSO proposed the use of a hybrid optimization along with a simplex method to 
identify delamination in laminated beams (Qian, Cao, Su, \& Chen, 2012). Han, Xu, Wang, and Tian (2014) suggest an Efficient Managing Particle Swarm Optimization (EMPSO) for high dimension problems to estimate defect profile from magnetic flux leakage signals. The experimental results demonstrated that the inversing technique based on EMPSO is capable of estimating 2-D defect profiles and outperforms the inverse technique. In the same way, Blanloeuil, Nurhazli, and Veidt (2016) propose using PSO for optimal sensor placement to improve the damage identification in an SHM approach. Santos et al. (2016) address a method based on Memetic algorithm along with PSO to improve the stability and reliability of the expectation-maximization algorithm applied to damage identification on bridges. Also, the chaotic PSO algorithm was used to determine support vector machine parameters for predicting model of dam displacement (Su, Wen, Chen, \& Tian, 2016). Summarizing the previously presented work, the majority of them have only focused on optimizing sensors placement or estimating/identifying somewhat specific within those methods instead of using to compute SFAN optimal setup parameters as proposed here.

The literature shows clearly that there is still not sufficient research on identifying structural damage progression in composite materials. Furthermore, it is evident that there is a lack of using a combination of Kappa-PSO and FAN along with EMI when applied to monitor the progress of structural damage in SHM systems. Thus the proposed method presents a novel SHM approach. It is also important to highlight that the research presented here is a continuation of previous authors work related to identifying damage growth by using SFAN (De Oliveira \& Inman, 2016, 2017). Unlike existing studies, a major contribution of this work consists of a new and reliable strategy for improving the identification of damage progression in composite structures, via SHM by focusing on using PSO based method to automatically select optimal FAN parameters. In order to evaluate the proposed methodology, experimental tests are conducted based on the EMI technique by using structural response signals in the time-domain. Validation of the proposed method is carried out in a unidirectional composite plate with four attached PZT patches. Damage growth scenarios are simulated by loosening bolts to three different levels. Also, the repaired structural condition is also considered by retightening the bolts. Therefore, the results show that it can identify various structural conditions with accuracy, reliability, and efficiency. Further, in order to demonstrate the superiority of the proposed methodology the results are compared with others recently proposed in literature.

In summary, the main contributions of this paper are:

- A method which takes advantage of the supervised learning of FAN by using PSO along with the EMI technique in order to get an enhanced performance compared to other work presented in the SHM literature. In the context of identifying the progression of structural damage several tools and techniques from intelligent diagnosis have been combined.

- A method to reduce the practical cost to select optimal parameters of the FAN classifier is presented. As a consequence, this method, which relies on concepts that the optimum setup parameters, is directly related to enhancing the success rate while the damage identification is being performed.

- The proposed method uses the Kappa coefficient as an objective function to be maximized through the PSO algorithm in order to automatically select the best FAN setup parameters.

The remainder of the paper is organized as follows: firstly, the main theoretical fundamentals are addressed. Secondly, the proposed method, which is based on using supervised learning of FAN via the Kappa-PSO algorithm, is presented. Subsequently, the experimental setup and results are presented followed by compar-
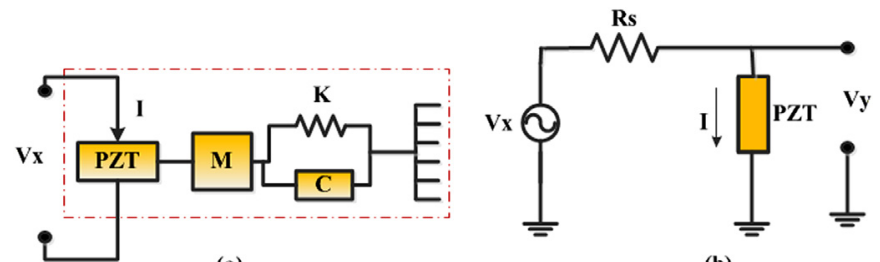

(a)

(b)

Fig. 1. (a) Electromechanical coupling between the PZT patch and the host structure; (b) circuit used for exciting the set PZT/structure.

ison with other state-of-the-art solutions. Finally, the paper concludes highlighting advantages and remarks of the proposed approach.

\section{Theoretical fundamentals}

\subsection{Time-domain analysis based on the Electromechanical Impedance}

A technique based on the Electromechanical Impedance (EMI) for SHM systems was originally proposed by Liang et al. (2014) and has been improved by several other authors (Annmandas \& Soh, 2010; Chaudhry, Lalande, Ganino, Rogers, \& Chung, 1996; Cortez, Vieira Filho, \& Baptista, 2012; Giurgiutiu \& Zagrai, 2002; Sun, Chaudhry, Liang, \& Rogers, 1995; Vieira Filho, Baptista, \& Inman, 2011; Yang, Liu, Annamdas, \& Soh, 2009; Zagrai \& Giurgiutiu, 2001). This technique is a Non-Destructive Evaluation (NDE) method which is based on the Frequency Response Function (FRF). The monitored structure, including the PZT, is represented by an electromechanical model of mass-spring with a single degree of freedom as presented in Fig. 1(a) (Liang et al., 2014). In Fig. 1(a), M, $\mathrm{C}$ and $\mathrm{K}$ represent mass, spring constant and damping coefficient respectively. Exciting the PZT using a sinusoidal source $V_{X}$ (with amplitude $\mathrm{V}_{\mathrm{xP}}$ and angular frequency $(\omega)$ ) will produce a current I with amplitude $I_{P}$ and phase $\Psi$. Thus, the electrical impedance of the PZT $\left(Z_{\mathrm{E}}(\omega)\right)$ is given as follows (Liang et al., 2014):

$\mathrm{Z}_{\mathrm{E}}(\omega)=\frac{\mathrm{Vx}}{\mathrm{I}}=\frac{1}{\mathrm{j} \omega \mathrm{a}}\left(\bar{\varepsilon}_{33}^{\mathrm{T}}-\frac{\mathrm{Z}(\omega)}{\mathrm{Z}(\omega)+\mathrm{Z}_{\mathrm{a}}(\omega)} \mathrm{d}_{3 \mathrm{x}}^{2} \hat{\mathrm{Y}}_{\mathrm{xx}}^{\mathrm{E}}\right)^{-1}$

where $Z_{a}(\omega)$ and $Z(\omega)$ represent the mechanical impedances for the transducer and monitored structure, respectively. In Eq. (1), $\bar{\varepsilon}_{33}^{T}, \hat{\mathrm{Y}}_{\mathrm{Xx}}^{\mathrm{E}}, d_{3 x^{\prime}}^{2} a$, and $j$ represent dielectric constant, Young's modulus, electric field constant, geometric constant and imaginary unit respectively. Note from Eq. (1) that any variation in terms of the structural impedance will cause changes into the electrical impedance of the PZT patch and this in turn causes changes in the EMI signatures.

In EMI-based techniques, the structure is excited in an appropriated frequency range through the attached PZTs, which are also used as sensors to capture the signal responses from the structure. In general, the results from these measurements are used to determine FRF and subsequently the EMI. Generally, statistical indices are computed from EMI signatures and then used to detect structural damage. The most common indices are Root Mean Square Deviation (RMSD), Correlation Coefficient Deviation Metric (CCDM) and Mean Absolute Percentage Deviation (MAPD). These indices are computed using signals obtained from the structure in healthy condition, known as baseline, as well as signals obtained from the structure under test. Further details about EMI in SHM systems are found in the follow references (Liang et al., 2014; Park et al., 2003).

Recently, then EMI technique has been developed through contributions on several fronts and applications including signal processing, statistical methods and new circuits for excitation/reception of signals from the PZT/structure. The proposed 
method uses the EMI principle, however the analysis is carried out in the time-domain without computing either FRF or the EMI. The EMI-based time-domain analysis is a recent development and was first presented in Vieira Filho et al. (2011). The authors proposed multilevel wavelet decomposition via time responses obtained from a piezoelectric wafer in an impedance-based method. The above work compares the EMI based on the Frequency Response Function (FRF) to the wavelet based method in the timedomain using RMSD and CCDM indices to detect structural damage. Cortez et al. (2012) and De Oliveira and Inman (2016, 2017) also provided progress in using EMI in the time-domain applied to SHM applications.

Time-domain analysis can be carried out considering the excitation circuit of the PZT/structure developed by Baptista and Vieira Filho (2009) and as presented in Fig. 1(b). Denoting a sinusoidal excitation signal $V_{X}$ with frequency $\omega$ and peak voltage $V_{x P}$, the circuit analysis can be mathematically represented in two ways. First, the traditional method based on the frequency response which requires the transfer function of the circuit is given by

$\frac{V_{y}(\omega)}{V_{x}(\omega)}=\frac{\mathrm{Z}}{\mathrm{Z}+R_{S}}$

where $Z$ represents the impedance of PZT/structure and $R_{S}$ is a precision resistor used to limit the current through the PZT. From the transfer function, the time response $V_{y}$ can be obtained through inverse Laplace or Fourier Transform considering the excitation signal $\mathrm{V}_{\mathrm{X}}$.

The second way uses the definition of PZT impedance, which is given by

$\mathrm{Z}(\omega)=\frac{\mathrm{V}_{\mathrm{y}}(\omega)}{\mathrm{I}(\omega)}$

where $\mathrm{Z}(\omega)$ represents the impedance of PZT/structure, $\mathrm{V}_{\mathrm{y}}(\omega)$ voltage, I $(\omega)$ the excitation current at the PZT and $\omega$ the angular frequency. Considering the phase $\Psi$, the impedance can be written as (Cortez et al., 2012)

$\mathrm{Z}(\omega)=\frac{\mathrm{V}_{\mathrm{y}}}{\mathrm{I}} \cos (\Psi)+\mathrm{j} \frac{\mathrm{V}_{\mathrm{y}}}{\mathrm{I}} \sin (\Psi)$

where $j$ represents the imaginary unit. Considering the resistor for limiting the current through $R_{S}$, the impedance can be written as follows (Cortez et al., 2012)

$\mathrm{Z}_{\mathrm{Re}}=\mathrm{R}_{\mathrm{s}} \frac{\mathrm{V}_{\mathrm{y}}}{\mathrm{V}_{\mathrm{x}}-\mathrm{V}_{\mathrm{y}}} \cos (\Psi)$

$\mathrm{Z}_{\mathrm{Im}}=\mathrm{R}_{\mathrm{s}} \frac{\mathrm{V}_{\mathrm{y}}}{\mathrm{V}_{\mathrm{x}}-\mathrm{V}_{\mathrm{y}}} \sin (\Psi)$

where $Z_{R e}$ and $Z_{I m}$ represent the real and imaginary parts of impedance, respectively. In general, the real part of the EMI presents higher sensitivity than the respective magnitude of the imaginary part for damage detection. Owing to that, many authors consider only the real part of the EMI for SHM applications (Park et al., 2003). From Eq. (5), if the excitation voltage and the resistor $R_{S}$ are kept constant (in phase and peak), the response voltage $\left(\mathrm{V}_{\mathrm{y}}\right)$ is directly correlated with the impedance variation. The same analysis can be carried out to the imaginary part of the impedance.

Another way to analyze this is to consider the modulus of the Electromechanical Impedance. From Eqs. (5) and (6), the modulus of Electromechanical Impedance $|\mathrm{Z}|$ is given by:

$|\mathrm{Z}| \cong \frac{\mathrm{V}_{\mathrm{y}}}{\mathrm{V}_{\mathrm{x}}-\mathrm{V}_{\mathrm{y}}} \mathrm{R}_{\mathrm{s}}$

Solving Eq. (7) for the relationship between the voltages yields

$\mathrm{Vy} \cong \frac{|\mathrm{Z}|}{|\mathrm{Z}|+\mathrm{R}_{\mathrm{S}}} \mathrm{Vx}$

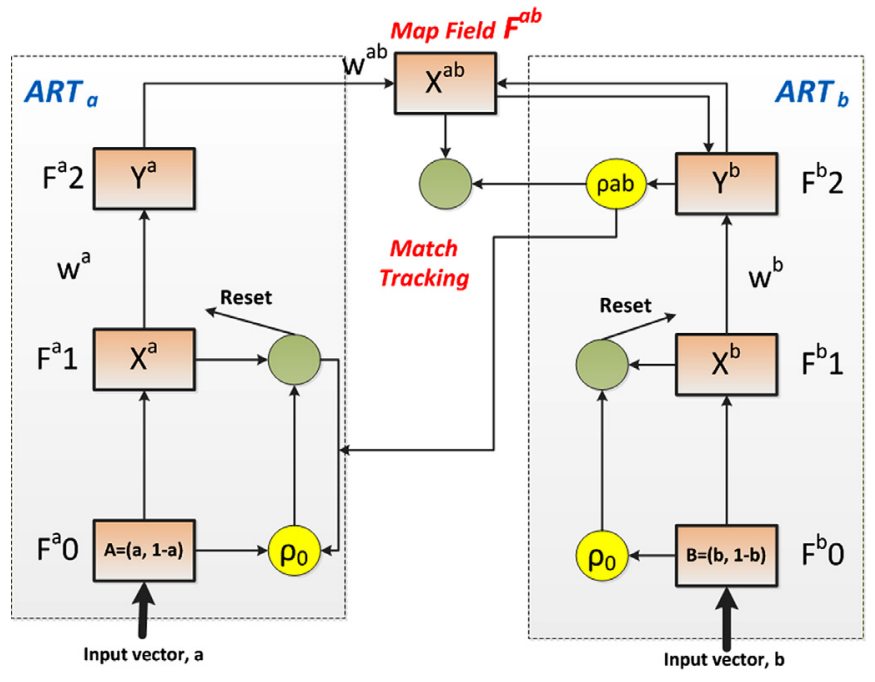

Fig. 2. Architecture of the Fuzzy ARTMAP neural network.

Analyzing Eqs. (5)-(8), it is clearly possible to identify structural damage using only the response signals of PZT/structure, because these signals are directly correlated with the Electromechanical Impedance (since it is guaranteed that the excitation input is kept constant).

Hence, in this work, the damage identification considers timedomain response $\left(\mathrm{V}_{\mathrm{y}}\right)$ only, without considering FRF or inverse Laplace/Fourier Transform, which by itself is a great advantage in terms of computational efforts. It simplifies and speeds up the damage identification in real applications. It is important to point out that EMI time-signals (Vy) are used as inputs to compute Euclidean distances throughout this approach.

\subsection{Fuzzy-ARTMAP network}

FAN architecture, which has a self-organizing learning network, was proposed by Carpenter, Grossberg, Markuzon, Reynold, and Rosen (1992). FAN belongs to the family of the adaptive resonance theory (ART) to avoid restarting the training of the classifier for each every input pattern, and so it allows for keeping and extending the previously obtained knowledge (Carpenter et al., 1992). The architecture of FAN comprises two fuzzy modules ARTa and ARTb, which have the same structure as the ART1 neural network, but uses fuzzy logic in its operation (Araújo et al., 2013; Carpenter et al., 1992). Also, the Map Field is responsible for linking ARTa with ARTb. Fig. 2 summarizes the architecture of the FAN network. Performance of the FAN depends critically on the fact that its setup parameters are chosen carefully in order to improve the classifier. The FAN parameters are described as follows. The choice parameter $(\alpha)$ defines the degree of interference on the selection of the most representative neurons of the weight vector. The training rate $(\beta)$ controls the speed at which the neural network learns. If $\beta$ is set to 1 the training time will be shorter. The vigilance parameter $(\rho)$ seeks to figure out the differences among input patterns that create new categories through similarity tests. The match tracking $(\varepsilon)$ checks if there is matching between the input (ARTa module) and output (ARTb module). If not, the vigilance parameter of ARTa is increased in order to search for another index that provides matching. If none of inputs existing in ARTa matches with the outputs of ARTb, then a new input is activated in ARTa which effects its connection with an output of ARTb. FAN is well-known in the literature and can be further explored in Carpenter et al. (1992). 


\subsection{Particle Swarm Optimization}

Particle Swarm Optimization (PSO) is a stochastic optimization technique based on population which was inspired by the social behavior of a flock of birds or school of fish (Kennedy \& Eberhart, 1995). This technique shares many similarities with evolutionary computational methods, such as genetic algorithms, although it does not contain evolutionary operators such as crossover and mutation. PSO belongs to a class of evolutionary computation algorithms that does not employ the concept of "law of the strongest", or the direct selection function. A solution with very low setting values can thus survive for optimizing and potentially go to any point in the solution space (Eberhart \& Shi, 1998). Unlike genetic algorithms which were designed to handle binary encoding, PSO was designed to provide effective results in solving global optimization problems in which the encoding values are real, thereby making PSO suitable for studies on larger scales.

Going a step further into the idea of PSO, each particle corresponds to a unique response in the solution space and population of particles, called a swarm. All particles are associated with positions, which are analyzed according to the evaluation function $(f p)$ to being optimized, and velocity values, which define their movements. Particles move through the solution space, following the particles with the best fit. Assuming a $d$-dimensional solution space, the position of the particle $i$ in the swarm containing Pparticles is represented by:

$\mathrm{S}_{\mathrm{i}}=\left(\mathrm{S}_{\mathrm{i} 1}, \mathrm{~S}_{\mathrm{i} 2}, \ldots \mathrm{S}_{\mathrm{id}}\right)$

where, $i=1,2, \ldots$. P. The velocity of the particle is referenced by the follow vector

$\mathbf{V}_{\mathrm{i}}=\left(\mathrm{V}_{\mathrm{i} 1}, \mathrm{~V}_{\mathrm{i} 2}, \ldots \mathrm{V}_{\mathrm{id}}\right)$

whereas the best previously visited position of the particle is defined as follows:

$\mathbf{P}_{\mathrm{i}}=\left(\mathrm{P}_{\mathrm{i} 1}, \mathrm{P}_{\mathrm{i} 2}, \ldots \mathrm{P}_{\mathrm{id}}\right)$

For each new iteration $q+1$, the velocity and position of the particle $i$ are updated according to equations bellow:

$\mathbf{V}_{\mathbf{i}}^{\mathbf{q}+1}=\mathbf{w}^{\mathbf{q}} \mathbf{V}_{\mathbf{i}}^{\mathbf{q}}+\mathrm{c}_{1} \mathrm{r}_{1}\left(\mathbf{P}_{\mathbf{i}}^{\mathbf{q}}-\mathbf{S}_{\mathbf{i}}^{\mathbf{q}}\right)+\mathrm{c}_{2} \mathrm{r}_{2}\left(\mathbf{P}_{\mathbf{g}}^{\mathbf{q}}-\mathbf{S}_{\mathbf{i}}^{\mathbf{q}}\right)$

$\mathbf{S}_{\mathbf{i}}^{\mathbf{q}+1}=\mathbf{S}_{\mathbf{i}}^{\mathbf{q}}+\mathbf{V}_{\mathbf{i}}^{\mathbf{q}+1}$

where $\boldsymbol{P}_{\boldsymbol{g}}$ represents the best global position of the particle in the swarm, $\boldsymbol{w}_{\boldsymbol{q}}$ is the inertial weight of the particle, $c_{1}$ and $c_{2}$ are positive constants known as cognitive and social parameters respectively, and $r_{1}$ and $r_{2}$ are random numbers evenly distributed in the interval of $[0,1]$. The $\boldsymbol{w}_{\boldsymbol{q}}$ regulates the balance between exploitation and use. A high weight facilitates a global search (holding), while a small value of weight tends to facilitate adjustment in a particular search area (use). This is because the values of the weights are defined as from a monotonically decreasing function of $q$. An appropriate tuning for $c_{1}$ and $c_{2}$ may result in a faster convergence of the algorithm and attenuation of the local minimum.

\section{Methodology}

The proposed methodology for identifying the progression of structural damage, by using Kappa-PSO based method to automatically selecting optimal FAN parameters, is presented in this section. In this sense, Fig. 3 summarizes the schematic diagram for the method. The methodology is done by means of three steps as described in the following subsections. In step 1, the impedance signals are obtained based on the EMI principle. For this, four PZTs (PZT \#1, PZT \#2, PZT \#3 and PZT \#4) considering five different structural conditions (Healthy (H), Level 1 (L1), Level 2 (L2), Level
3 (L3) and Repaired (R)), for each damage position (P1, P2, P3, $\mathrm{P} 4$ and P5) were considered. More details about the experimental setup are presented in the Section 4. In step 2, Euclidean distances are computed from the structural response signals. Euclidean distances are used as inputs for the FAN algorithm. In step 3, the PSO method is carried out aiming to obtain the optimal FAN parameters. For that, the Kappa coefficient is used as an objective function to be maximized in order to improve damage identification rates.

\subsection{Step 1: structural response signals}

The procedure of exciting the structure is carried out using a chirp signal for each PZT. The corresponding impedance signal for each PZT patch is then sampled separately. In this procedure, each PZT transducer acts as actuator and sensor at the same time. This procedure is well-known in EMI literature and it can be further explored in Liang et al. (2014) and Park et al. (2003). Impedance signals, for all PZTs patches, are obtained considering the pristine structural condition, after each simulated damage has been added to a structure and finally when all simulated damage is removed (repaired structural condition). Subsequently in the Section 4 is shown further details of the experimental setup used to obtain all impedance signals as well as the damage scenarios.

\subsection{Step 2: computing Euclidean distances}

This approach compute Euclidean Distances (ED) from the obtained impedance signals, using the developed MatLab ${ }^{\circledR}$ software, taking into account the signals of the healthy structural condition (baseline) as a reference by the following (De Oliveira \& Inman, 2016, 2017):

$\mathrm{L} 1(\mathrm{BL}, \mathrm{L} 1)=\sqrt{\sum_{j=1}^{n}\left(B L_{j}-L 1_{j}\right)^{2}}$

where, BL and L1 represent the healthy (baseline) and damaged structural (1/4 turn) conditions respectively. For the other structural conditions ( $\mathrm{H}, \mathrm{L} 2, \mathrm{~L} 3$ and $\mathrm{R}$ ) the same procedure is also carried out. A similar procedure is applied to each PZT patch and damage positions (P1, P2, P3, P4 and P5). Computed EDs are used to form the dataset for training and testing phases. This dataset is used to feed the FAN classifier.

\subsection{Step 3: supervised learning of FAN by using the Kappa-PSO algorithm}

Under this approach, the ARTa module is fed with the EDs values, which are computed from the obtained structural impedance signals, while the ARTb module has five recognition categories which are associated with five structural conditions (H, L1, L2, L3 and R). Both modules are linked by a Map Field module (Fab) which associates inputs of the ARTa with outputs of the ARTb by using the match tracking procedure in order to maximize the correct classification for identifying of structural damage (Fig. 2). Owing to the four FAN parameters (choice $(\alpha)$, training $(\beta)$, vigilance $(\rho)$ and match tracking $(\varepsilon)$ ) have substantial influence on FAN performance; therefore, instead of using an expert to selecting optimal parameters we propose using the Kappa-PSO algorithm to obtain automatically these optimal parameters as shown in the following.

For that, the PSO algorithm, explained previously, considers each particle as a FAN with a 4-dimensional solution space, where each dimension represents an indicator of optimal setup parameters required for classifying of various structural conditions. Thus, the components of the particle position $i$ in the solution space are defined, based on Eq. (9), by the following:

$S_{i}^{q}=\left(S_{11}^{q}, S_{12}^{q}, S_{13}^{q}, S_{14}^{q}\right)$ 


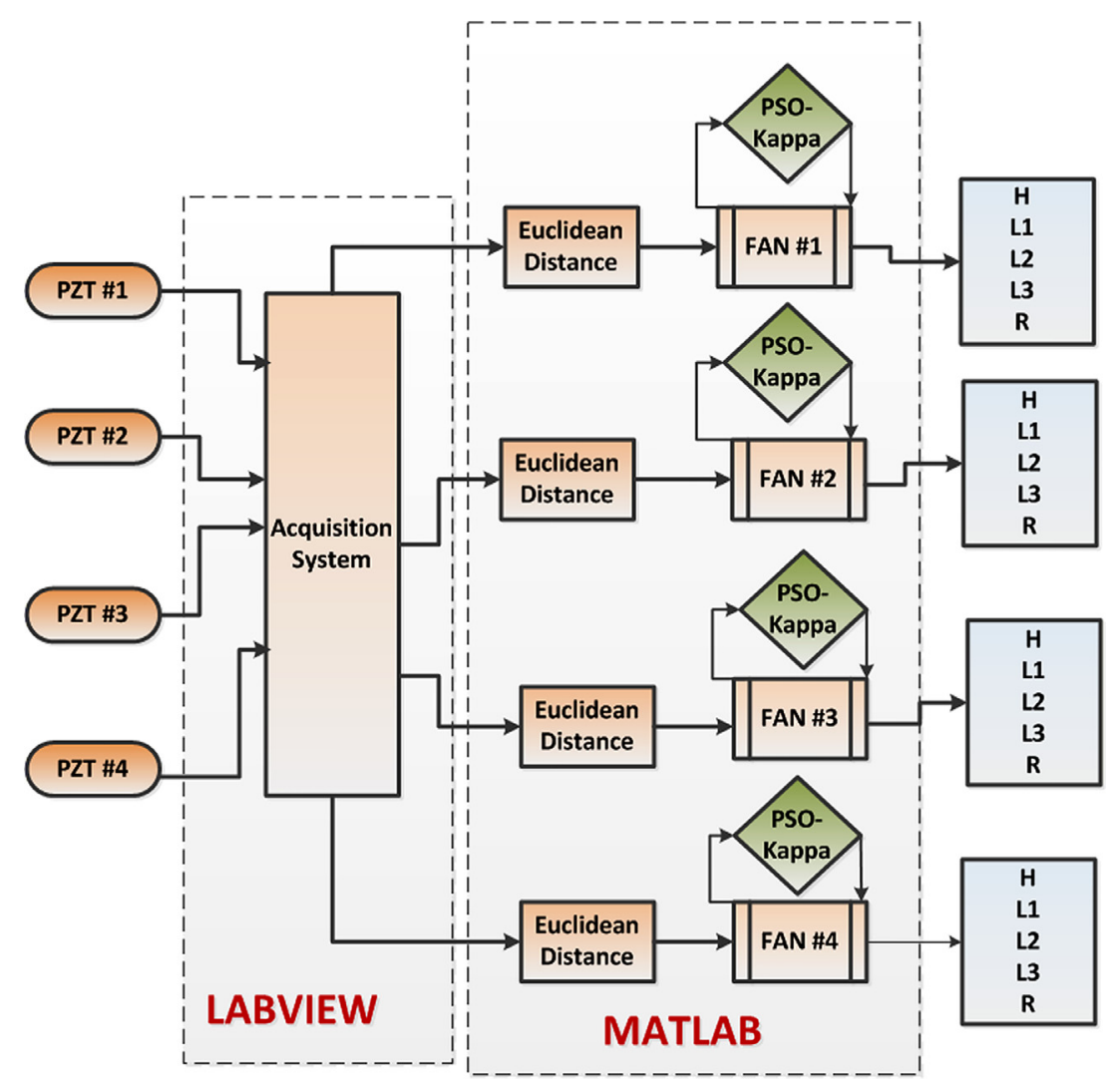

Fig. 3. Summarized schematic diagram for the proposed methodology.

here,

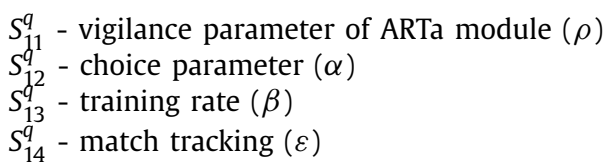

It is important to point out that the choice of these parameters for composing the particle position is essential to the evolution of the FAN (Carpenter et al., 1992; Carpenter, Grossberg, \& Rosen, 1991; Granger et al., 2007). In this approach, the Kappa coefficient is used as the objective function by the PSO algorithm to assess the quality of the particles. The procedure follows the guidelines summarized in Fig. 4, which requires establishing this optimal parameters setup for the FAN classifier. Under this approach, qmax indicates the maximum number of iteration in which the optimization process will run; $P$ is the population size of particles; Vmax indicates the maximum velocity that particles can move themselves; $w_{0}$ means initial inertial weight; $c_{1}$ and $c_{2}$ are positive constants that represent cognitive and social parameters, respectively; $r_{1}$ and $r_{2}$ are random numbers uniformly distributed in a range of $[0,1]$. Extra details about PSO along with FAN are found in Eberhart and Shi (1998) and Granger et al. (2007).

Essentially this approach proposes using FAN as the damage classifier algorithm along with the PSO metaheuristic algorithm which is used to determine an optimum parameter configuration that maximizes the identification rate of structural damage in SHM systems. As mentioned, the Kappa coefficient is also used as the objective function by the PSO algorithm to assess the quality of the particles. The Cohen's Kappa coefficient is a metric of agreement introduced firstly to the area of psychology in Cohen (1960). The original intention of Kappa was to measure the level of agreement or disagreement of a group of people observing the same phenomenon. In practice, both success rate and Kappa are com- puted from the confusion matrix. However, the main difference between them is that the success rate is only computed by using the principal diagonal of the confusion matrix whereas Kappa analyses the whole matrix. In this approach, we have a confusion matrix $\mathbf{M}$ with five rows and five columns. Hence, the Kappa coefficient $(\mathrm{K})$, which measures the observed proportional agreement (Po) between the existing and predicted classes over the training basis after removing the proportion of coincidence by chance (Pe), is defined as follows:

$$
\begin{aligned}
& \mathrm{K}=\frac{\mathrm{Po}-\mathrm{Pe}}{1-\mathrm{Pe}} \\
& \mathrm{Po}=\frac{\mathrm{TH}+\mathrm{TL} 1+\mathrm{TL} 2+\mathrm{TL} 3+\mathrm{TR}}{\mathrm{N}} \\
& \mathrm{Pe}=\frac{(\mathrm{C} 1 * \mathrm{~L} 1)+(\mathrm{C} 2 * \mathrm{~L} 2)+(\mathrm{C} 3 * \mathrm{~L} 3)+(\mathrm{C} 4 * \mathrm{~L} 4)+(\mathrm{C} 5 * \mathrm{~L} 5)}{\mathrm{N}^{2}}
\end{aligned}
$$

here,

TH- Number of $\mathrm{H}$ samples classified as true;

TL1- Number of L1 samples classified as true;

TL2- Number of L2 samples classified as true;

TL3- Number of L3 samples classified as true;

TR- Number of R samples classified as true;

C1- Number of H, L1, L2, L3 and R samples classified as H;

C2- Number of H, L1, L2, L3 and R samples classified as L1;

C3- Number of H, L1, L2, L3 and R samples classified as L2;

C4 - Number of H, L1, L2, L3 and R samples classified as L3;

C5- Number of H, L1, L2, L3 and R samples classified as R;

L1- Number of $\mathrm{H}$ samples classified as $\mathrm{H}$, or L1, or L2, or L3 or $\mathrm{R}$;

L2 - Number of L1 samples classified as H, or L1, or L2, or L3 or $\mathrm{R}$; 


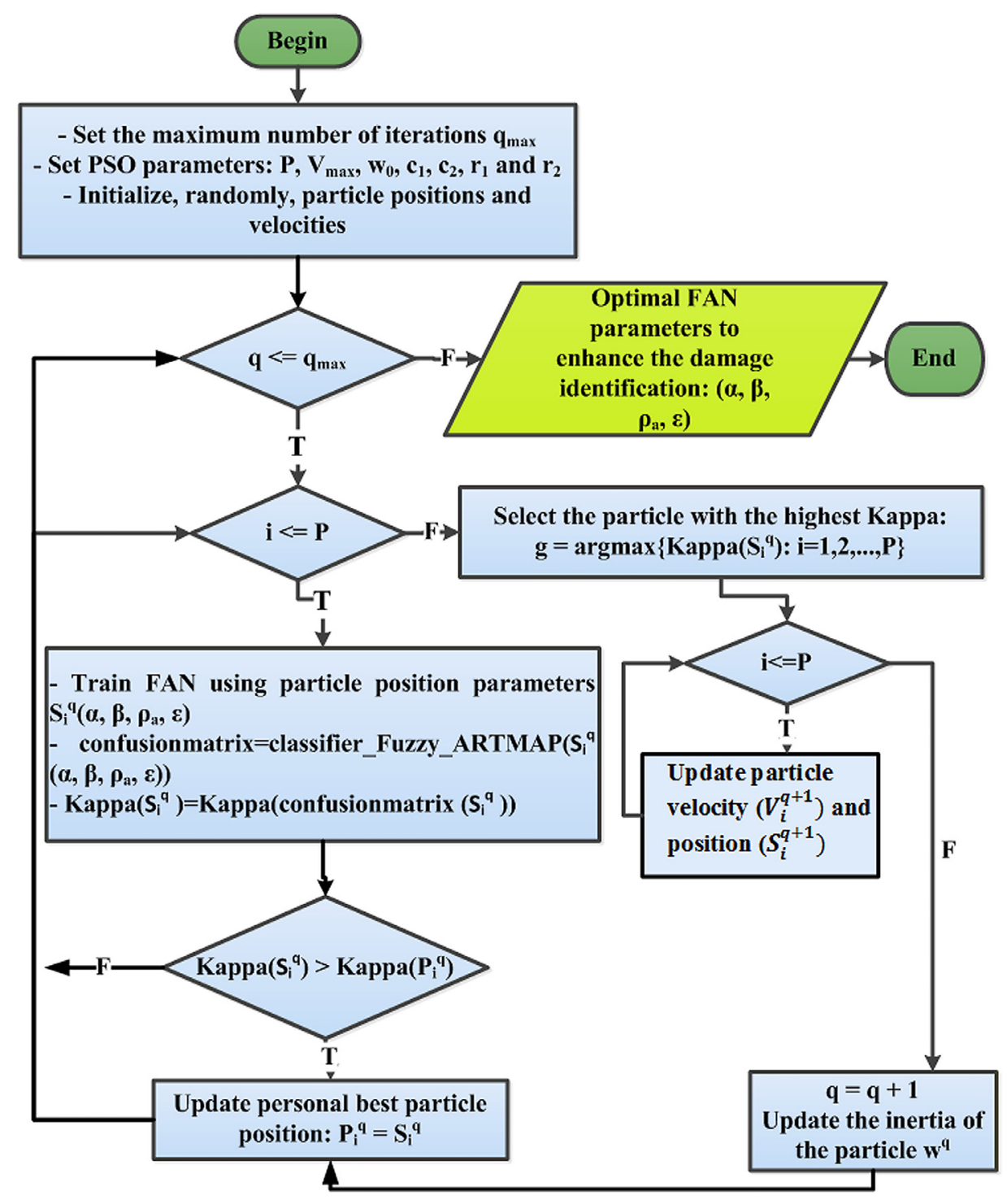

Fig. 4. Flowchart containing the whole procedure used to selecting the FAN optimal parameters by using the Kappa-PSO algorithm.

L3- Number of L2 samples classified as H, or L1, or L2, or L3 or $\mathrm{R}$;

L4 -Number of L3 samples classified as $\mathrm{H}$, or L1, or L2, or L3 or $\mathrm{R}$;

L5- Number of R samples classified as $\mathrm{H}$, or L1, or L2, or L3 or $\mathrm{R}$;

$\mathrm{N}$ - Total of classified samples.

It is important to point out that $\mathrm{H}, \mathrm{L} 1, \mathrm{~L} 2, \mathrm{~L} 3$ and $\mathrm{R}$ refer to Euclidean distances computed considering the following structural conditions: Healthy, damaged (Level 1, Level 2, Level3) and Repaired, respectively.

In short, the interpretation of $\mathrm{K}$ can be made as follows: the nearest to zero is $\mathrm{K}$, it means that the sorted units occurred by chance, on the other hand when $\mathrm{K}$ approaches 1 , the agreement between the correct classes and predicted tends to the "exact" (Cohen, 1960).

\section{Experimental setup}

In order to evaluate the proposed methodology, experimental tests were carried out as follows. Firstly, a composite plate was attached to an aluminum table which has twelve threads at differ- ent points using also twelve sets composed of bolts and washers. The plate was only in contact with the table at those twelve points through the washers. The thickness of each washer is $2 \mathrm{~mm}$ and its diameter is $6 \mathrm{~mm}$. The composite plate (ACP Composites) consists of a carbon fiber unidirectional pre-impregnated with epoxy resin of size $500 \mathrm{~mm} \times 300 \mathrm{~mm} \times 1.5 \mathrm{~mm}$. Secondly, four piezoceramic disks (PSI-5H4E/Piezo Systems), called PZT \#1, PZT \#2, PZT \#3 and PZT \#4, were bonded to the composite plate at different distances as shown in Fig. 5. Each PZT has external diameter of $31.8 \mathrm{~mm}$.

Experimental tests were conducted by using the acquisition system proposed in Baptista and Vieira Filho (2009). Their system was developed on the LabVIEW $^{\circledR}$ platform and makes use of a National Instruments Data Acquisition (DAQ) device, model USB6259 (Figs. 5 and 6). The acquisition system is responsible to excite the structure using a chirp signal varying from $20 \mathrm{kHz}$ to $180 \mathrm{kHz}$. The amplitude of excitation, for each PZT, was also swept through five different voltage levels: $1 \mathrm{~V}, 2 \mathrm{~V}, 3 \mathrm{~V}, 4 \mathrm{~V}$ and $5 \mathrm{~V}$. Resistors $\mathrm{R}$ are used to limit the current through PZTs and they were set to $1 \mathrm{k} \Omega$. Further details about the experimental setup are found in De Oliveira and Inman (2016, 2017).

The structure was excited considering the pristine condition and its correspondent signal for each PZT was stored as the base- 


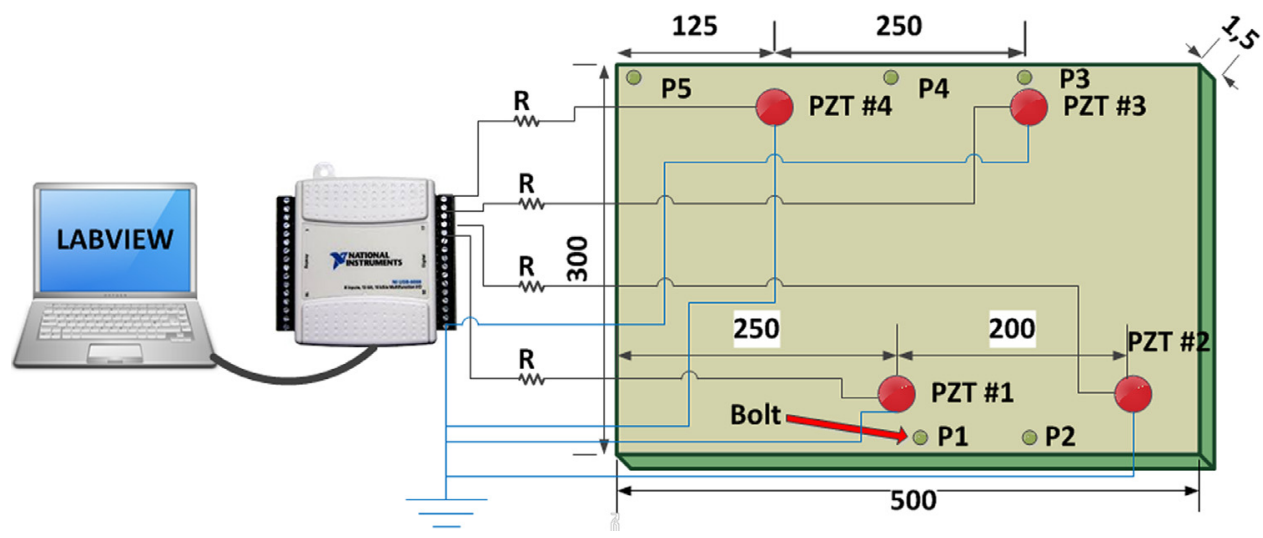

Fig. 5. Representation of the general diagram for the acquisition system (dimensions in millimeters).

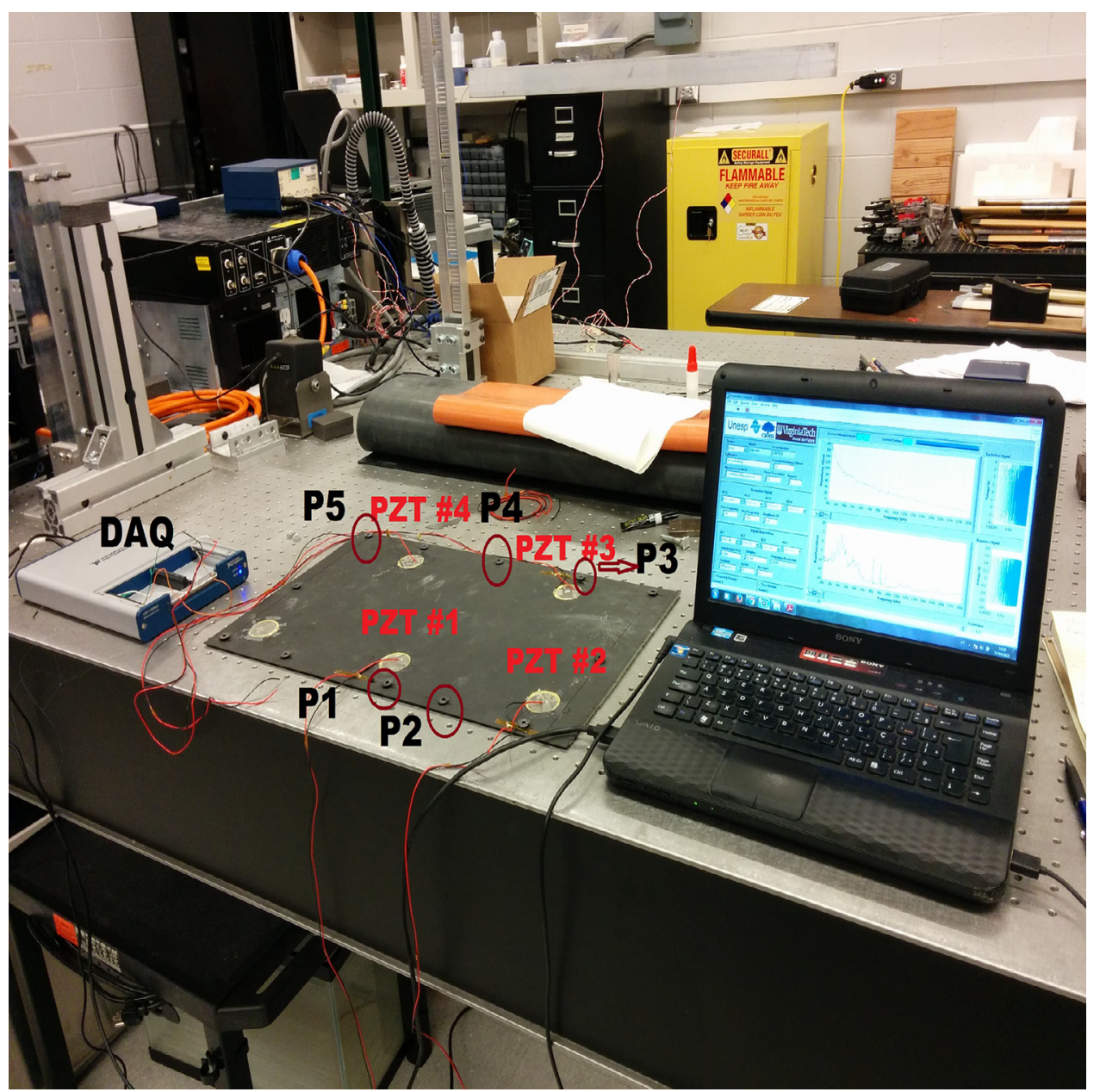

Fig. 6. Experimental set up including: the composite plate containing four PZTs patches, DAQ and laptop running the acquisition software.

line (BL). At a different time, a second dataset was also obtained considering the pristine structural condition (called H). Posteriorly, structural damage was simulated by loosening bolts at three different levels called L1, L2 and L3. The levels L1, L2 and L3 represent the progression of structural damage due to loosening bolts by a $1 / 4,1 / 2$ and $1 / 1$ turns respectively. Afterwards, the repaired structural condition was also evaluated by retightening bolts (R). This procedure was carried out for all positions P1, P2, P3, P4 and P5 by considering all five different levels of excitation signals.

\section{Experimental results}

In order to evaluate the proposed methodology, this section presents the experimental results. Firstly, structural response signals were obtained considering various structural conditions, positions of damage and PZTs (using the aforementioned acquisition system). Fig. 7 present some of the data. Fig. 7(a) depicts the real part of the impedance for PZT \#1 and position P1. Signatures are shown for four different structural conditions: healthy $(\mathrm{H})$, level L1 (1/4 turn), level L2 (1/2 turn) and level L3 (1/1 turn). As observed, change in the impedance signatures caused by damage insertion 


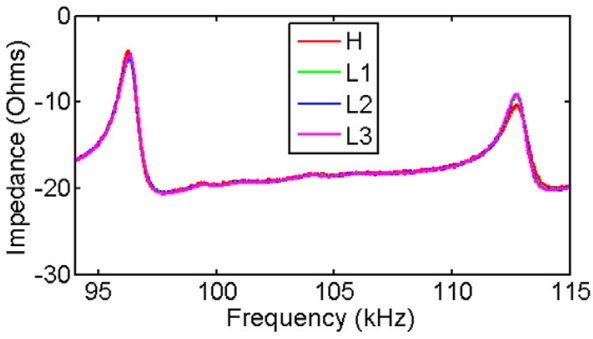

(a)

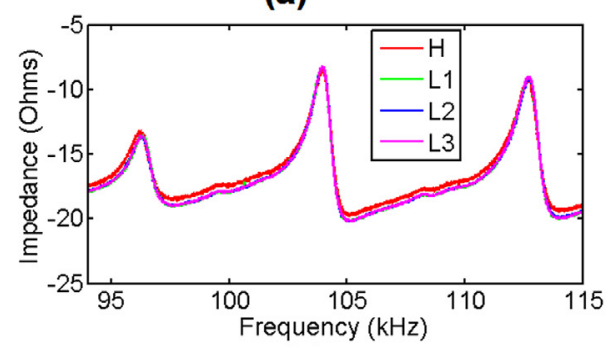

(c)

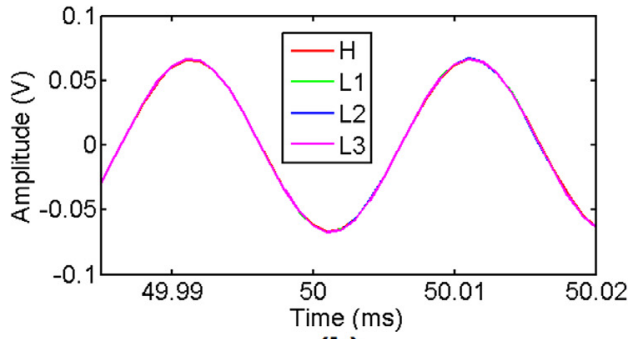

(b)

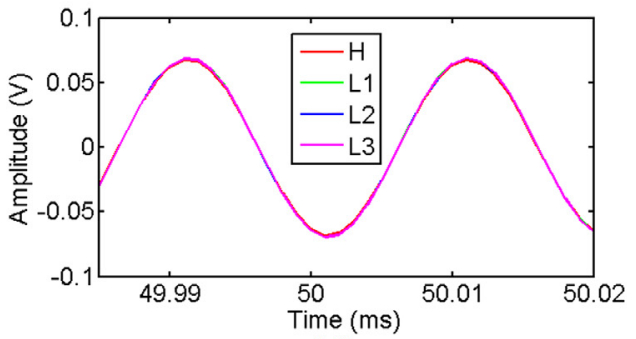

(d)

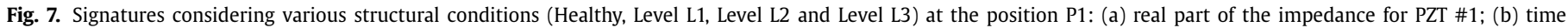
responses for PZT \#1; (c) real part of the impedance for PZT \#2; (d) time responses for PZT \#2.

Table 1

Distribution of EDs at position P1 into datasets for PZTs \#1 and $\# 2$.

\begin{tabular}{llllll}
\hline Structural conditions & \multicolumn{2}{l}{ PZT \#1 } & & & PZT\#2 \\
\cline { 2 - 3 } \cline { 5 - 6 } & Training & Test & & Training & Test \\
\hline Healthy (H) & 15 & 10 & & 15 & 10 \\
Level L1 & 15 & 10 & & 15 & 10 \\
Level L2 & 15 & 10 & & 15 & 10 \\
Level L3 & 15 & 10 & & 15 & 10 \\
Repaired (R) & 15 & 10 & & 15 & 10 \\
Total & 75 & 50 & & 75 & 50
\end{tabular}

is extremely subtle even for the position $\mathrm{P} 1$, which is placed very close to PZT \#1. Fig. 7(b) shows the corresponding structural time responses. Once again, changes caused by damage are almost imperceptive. In the same way, Fig. $7(\mathrm{c})$ and (d) present the real part of the impedance and structural time response respectively, under the same conditions, for PZT \#2. PZT \#2 is placed almost $200 \mathrm{~mm}$ far away from position P1 and the changes caused by damage are also extremely subtle. Despite the structural change being subtle, the proposed method (Kappa-PSO-FAN) overcomes this limitation, thereby improving the damage identification procedure as presented in the following.

Secondly, Euclidean distances were computed from the obtained response signals, in the time domain, as in Eq. (14). EDs are used to form dataset for training and testing phases, which are used as input to the FAN classifier. Table 1 summarizes datasets for structural conditions related to the position P1 considering PZT \#1 and PZT \#2. Similar datasets were also performed for other damage positions (P2, P3, P4 and P5) and PZTs (PZT \#3 and PZT \#4).

Thirdly, datasets are used as input to the FAN classifier. Four FAN (one for each PZT) were implemented for position P1 to analyze various structural conditions. A similar procedure was carried out for other damage positions (P2, P3, P4 and P5). FAN setup parameters, $\rho=0.78, \alpha=0.25, \beta=1$ and $\varepsilon=0.01$, were considered for both training and testing procedures. Setup parameters were chosen according to De Oliveira and Inman (2017). It is important to mention that the method codes the training labels, for all methods presented here, as 1 for $\mathrm{H}, 2$ for L1, 3 for L2, 4 for L3 and 5 for R. The output test is also labeled as 1, 2, 3, 4 and 5 for H, L1,
Table 2

Overall success rates for FAN and Kappa-PSO-FAN methods, considering four PZTs and all damage positions.

\begin{tabular}{llll}
\hline Positions & PZTs & FAN & Kappa-PSO-FAN \\
\hline P1 & PZT \#1 & $98 \%$ & $100 \%$ \\
& PZT \#2 & $50 \%$ & $56 \%$ \\
& PZT \#3 & $70 \%$ & $74 \%$ \\
P2 & PZT \#4 & $82 \%$ & $84 \%$ \\
& PZT \#1 & $82 \%$ & $86 \%$ \\
& PZT \#2 & $68 \%$ & $84 \%$ \\
& PZT \#3 & $70 \%$ & $72 \%$ \\
P3 & PZT \#4 & $58 \%$ & $70 \%$ \\
& PZT \#1 & $80 \%$ & $86 \%$ \\
& PZT \#2 & $56 \%$ & $70 \%$ \\
P4 & PZT \#3 & $100 \%$ & $100 \%$ \\
& PZT \#4 & $84 \%$ & $84 \%$ \\
& PZT \#1 & $\mathbf{4 6 \%}$ & $\mathbf{7 4 \%}$ \\
& PZT \#2 & $42 \%$ & $52 \%$ \\
P5 & PZT \#3 & $\mathbf{4 0 \%}$ & $\mathbf{6 0 \%}$ \\
& PZT \#4 & $\mathbf{6 8 \%}$ & $\mathbf{8 8 \%}$ \\
& PZT \#1 & $56 \%$ & $60 \%$ \\
& PZT \#2 & $32 \%$ & $54 \%$ \\
& PZT \#3 & $68 \%$ & $68 \%$ \\
& PZT \#4 & $74 \%$ & $75 \%$ \\
\hline
\end{tabular}

L2, L3 and R respectively. Damage identification is carried out off line and then the success rate for each structural condition/PZT is automatically computed.

As a result, Table 2 summarizes the success rates for FAN considering all positions of structural damage. Here, the success rates are considered as the ability of the classifier to distinguishing various structural conditions: Healthy (H), Level L1 (1/4 turn), level L2 (1/2 turn), level L3 (1/1 turn) and repaired (R). For example, FAN obtained a rate of $98 \%$ for PZT \#1 when the damage was placed at position P1, which means that our system was successfully able to identifying all five classes of structural conditions with $98 \%$ of accuracy. As showed in Fig. 5, the damage position P1 is placed close to the PZT \#1.

When the damage is far away from PZT patches, it is expected that the success rate of the classifier tends to decrease proportionally due to the drastic reduction of change in the structural conditions caused by damage. Hence, the success rate for position P2, 
Table 3

Obtained PSO parameters after the optimization process has been performed.

\begin{tabular}{ll}
\hline Parameters & Values \\
\hline Number of iterations $(\mathrm{q})$ & 100.00 \\
Number of population particles $(\mathrm{P})$ & 5.00 \\
Initial inertial weight $\left(\mathrm{w}_{0}\right)$ & 0.40 \\
Random parameter $\left(\mathrm{r}_{1}\right)$ & 0.02 \\
Random parameter $\left(\mathrm{r}_{2}\right)$ & 0.68 \\
Cognitive $\left(\mathrm{c}_{1}\right)$ and social $\left(\mathrm{c}_{2}\right)$ parameters & 2.00 \\
\hline
\end{tabular}

which is placed in a half way between PZT \#1 and PZT \#2, hit 82\% and $68 \%$ respectively. PZT \#3 was able to identifying all structural conditions with $100 \%$ of accuracy (for P3). This result was expected because the damage position (P3) is placed just a few millimeters from PZT \#3. Analyzing the results for position P4, it can be clearly noticed that the best result was obtained by PZT \#4 which hit an accuracy rate of the $68 \%$. Unfortunately, the proposed method was not able to identify those structural conditions with precision (over $70 \%$ ). Finally, the results for position P5 showed that PZT \#4 was able to identify all structural conditions with $74 \%$ of accuracy.

As mentioned, the best setup parameter for FAN is essential for enhancing the success rates of the classifier. For this reason, the Kappa-PSO-FAN algorithm is proposed here. Hence, the KappaPSO algorithm was run and the obtained PSO setup parameters are presented in Table 3. Also, optimal FAN parameters were computed automatically by using the PSO algorithm and used for both training and testing procedures. For example, obtained parameters for PZT \#1 and position P1 are: $\rho=0.0097, \alpha=0.0524, \beta=1$ and $\varepsilon=0.1438$. The number of Kappa-PSO-FAN networks is also the same that was used for FAN (Fig. 3).

The results obtained for the Kappa-PSO-FAN based method, considering the same structural conditions used for the FAN algorithm, are also presented in Table 2. As observed in Table 2, the success rates for the majority of the PZTs and damage positions have significantly increased when using the Kappa-PSO-FAN algorithm. Special highlight can be given for the obtained results for PZTs \#1, PZTs \#3 and \#4, when damage were applied to position P4, which presented improvements about $20 \%$ plus. Overall, the success rates hit at least $70 \%$ for those PZTs at which are more propitious to identifying structural damage being located closer to the damage positions. As a result, Kappa-PSO-FAN gave us an additional potential for further development of the methodology towards more precise damage localization and severity evaluation.

\section{Comparison with other state-of-the-art solutions}

In order to demonstrate the enhancement of the proposed method over the other existing methods in literature, we address a comparison among the proposed method with two others. It is noteworthy to mention that there are only two methods which handle with the identification of progression of structural damage in composite by using neural networks along with EMI (De Oliveira \& Inman, 2016, 2017). In order to have a fair comparison, all methods are applied to the same datasets, computer setup and conditions. Hence, Table 4 shows a comparison among the proposed methods (FAN and Kappa-PSO-FAN) and those ones proposed in De Oliveira and Inman (2016) (SFAN) and De Oliveira and Inman (2017) (PNN). For the purpose of brevity, only the most significant results are shown. It is important to clarify that the adopted SFAN setup parameters were: $\rho=0.78, \alpha=0.25$ and $\beta=1$ (De Oliveira \& Inman, 2017). For PNN, the spread constant $(\sigma)$ was set to 0.1 (De Oliveira \& Inman, 2017). Setup parameters for FAN were the same as proposed in the last section.
Table 4

Comparison for overall success rates for five different methods considering four PZTs and all damage scenarios. Methods based on PNN and SFAN were addressed in De Oliveira and Inman (2017) and De Oliveira and Inman (2016) respectively.

\begin{tabular}{llllll}
\hline Positions & PZTs & PNN & SFAN & FAN & Kappa-PSO-FAN \\
\hline P1 & PZT \#1 & $96 \%$ & $98 \%$ & $98 \%$ & $100 \%$ \\
& PZT \#4 & $62 \%$ & $82 \%$ & $82 \%$ & $84 \%$ \\
P2 & PZT \#1 & $72 \%$ & $82 \%$ & $82 \%$ & $86 \%$ \\
& PZT \#2 & $68 \%$ & $76 \%$ & $76 \%$ & $84 \%$ \\
P3 & PZT \#3 & $80 \%$ & $100 \%$ & $100 \%$ & $100 \%$ \\
& PZT \#4 & $60 \%$ & $84 \%$ & $84 \%$ & $84 \%$ \\
P4 & PZT \#3 & $\mathbf{6 4 \%}$ & $\mathbf{4 0 \%}$ & $\mathbf{4 0 \%}$ & $\mathbf{6 0} \%$ \\
& PZT \#4 & $\mathbf{7 8 \%}$ & $\mathbf{6 8} \%$ & $\mathbf{6 8} \%$ & $\mathbf{8 8} \%$ \\
P5 & PZT \#3 & $48 \%$ & $68 \%$ & $68 \%$ & $68 \%$ \\
& PZT \#4 & $72 \%$ & $70 \%$ & $74 \%$ & $75 \%$ \\
\hline
\end{tabular}

Table 5

Comparison among total times for: PNN, SFAN, FAN and Kappa-PSO-FAN.

\begin{tabular}{lll}
\hline References & Methods & Total time (ms) \\
\hline De Oliveira and Inman (2017) & PNN & 755.500 \\
De Oliveira and Inman (2016) & SFAN & 20.336 \\
Proposed here & FAN & 21.350 \\
Proposed here & Kappa-PSO-FAN & 9620.000 \\
\hline
\end{tabular}

Investigating the results presented in Table 4, it can be observed that the PNN based method presented the worst performance by taking into account the success rate for identifying structural damage. It is important to mention that PNN based methods have been widely recurrent in the SHM literature (De Oliveira \& Inman, 2017; Na \& Lee, 2013; Palomino et al., 2014; Selva et al., 2013). As observed, results for SFAN and FAN have presented practically the same performance for the overall success rate when working in the same conditions. On the other hand, there was a significant improvement for SFAN/FAN algorithms when compared with PNN for the majority of the cases analyzed. The last column in Table 4 presents the results considering the Kappa-PSO-FAN algorithm. It is worth noting that the success rates were significantly improved over other methods. Special highlight can be given for the obtained results, considering position P4, for PZTs \#3 and \#4 (highlighted in bold). Results were tremendously enhanced (about $20 \%$ plus) when compared with others. The PNN method can be further explored in Specht (1998).

Another interesting metric consists of the total (training + testing) time for all methods. Hence, Table 5 presents a comparison for all methods taking into account results for PZT\#1 and position P1. Similar results are found for the other cases.

As observed from Table 5, SFAN method presented the best performance in terms of the total time. This is no surprise that it proved advantageous due to the fact of the SFAN algorithm runs a step ahead of FAN in reducing the computational overhead and architectural redundancy which slows down the training of the network (Kasuba, 1993; Vakil-Baghmisheh \& Pavesic, 2003). In short, SFAN consists of an optimized, faster and simplified method of the original FAN. The FAN method obtained a second place with a subtle difference in relation to SFAN, followed by PNN. As expected, Kappa-PSO-FAN took almost $10 \mathrm{~s}$ when considered the same conditions as used for FAN, SFAN and PNN. The reason is that the PSOFAN method runs 100 iterations for each one of the 5 population particles (Table 3 ).

In order to investigate further time consumption, Table 6 depicts the influence of the number of iterations on the total time (training + test) and success rate for the Kappa-PSO-FAN method. For the purpose of brevity, only the results for P5 and PZT \#4 are presented. Analyzing Table 6 , it is clearly evident that the influence of the increase in the number of iterations is totally insignificant 


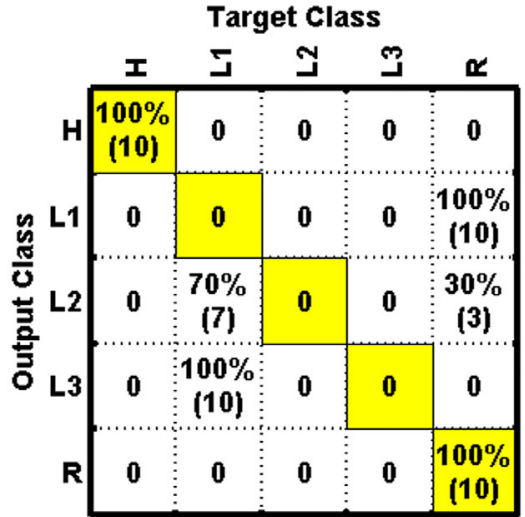

(a)

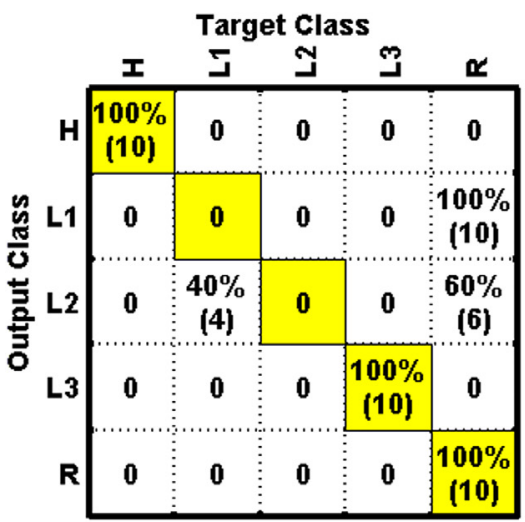

(c)

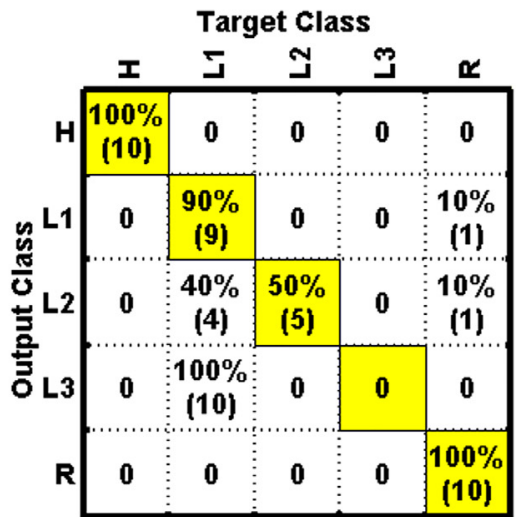

(b)

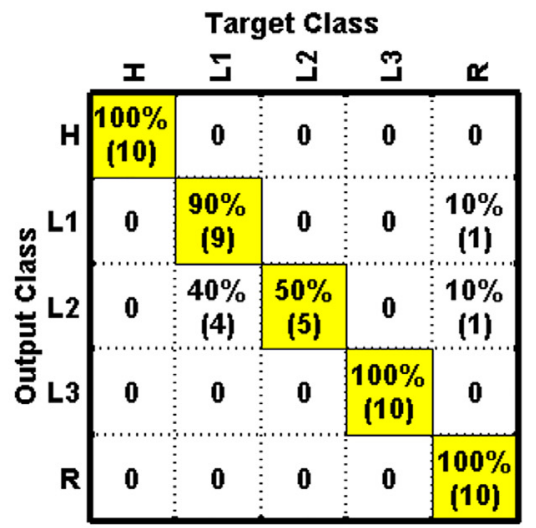

(d)

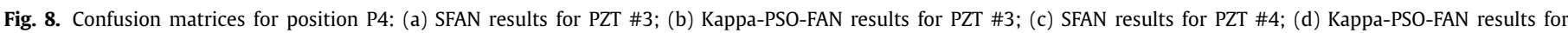
PZT \#4.

Table 6

Influence of the number of iterations on the total time and success rate while using the Kappa-PSO-FAN method (P5 and PZT \#4).

\begin{tabular}{lll}
\hline Number of iterations $(\mathrm{q})$ & Total time $(\mathrm{s})$ & Success rate $(\%)$ \\
\hline 10 & 1.040 & 75 \\
50 & 5.390 & 75 \\
100 & 7.770 & 75 \\
500 & 44.480 & 75 \\
1000 & 76.060 & 75 \\
\hline
\end{tabular}

for the success rate while the damage identification is being carried out. Meanwhile, the number of iteration is strictly related to the total time. It is clear that increasing the number of iterations for each one of the 5 population particles will cause a noticeable expenditure time resources. It is noticeable that choosing an appropriate tuning for $c 1$ and $c 2$ may result in a faster convergence of the algorithm and also reduce the local minimum.

In practice, the confusion matrix is considered a useful tool for analyzing how well the classifier can recognize features of different groups. A comparison between SFAN (De Oliveira \& Inman, 2016) and Kappa-PSO-FAN method is addressed in Fig. 8. For the purpose of brevity, only the most significant results are present (for PZTs \#3 and \#4 and position P4). As observed from Fig. 8(a), SFAN based method, for PZT \#3, was only able to identify two structural conditions ( $\mathrm{H}$ and $\mathrm{R}$ ), thereby rendering a very poor result. Fig. 8(b) presents the results for the Kappa-PSO-FAN algorithm. As observed, the results were enhanced especially for L1. On the other hand, the structural condition L3 was not identified. Fig. 8(c) de- picts the results for SFAN by using the PZT \#4. It is notable that this PZT was able to identify successfully only three different structural conditions (H, L3 and R). After applying the Kappa-PSO-FAN method, the results were overwhelmingly improved (Fig. 8(d)). It is clear that at least four structural conditions (H, L1, L3 and R) were successfully identified, whereas L2 was identified in $50 \%$ of the cases. Clearly, it is an undisputed fact that the method presented here was effectively able to successfully identify various structural conditions. Hence, one of the more significant findings to emerge from this study is that this can lead to a novel and efficient SHM method, based on Electromechanical Impedance principle along with Kappa-PSO-FAN, which is also shown to be sensitive enough to identify the progression of the structural damage.

\subsection{Advantages and drawbacks}

The feasibility of the proposed approach is validated based on EMI-measurement datasets. The advantages of the proposed method can be summarized into two points. Firstly, a novel way of automatically choosing the FAN setup is proposed. The architecture is inspired by PSO and Kappa. The method has direct implications in terms of diminishing the time consumption when choosing the best FAN setup parameters: choice, match tracking, vigilance and training. State-of-the-art approaches have manually handled the FAN-parameters selection (many attempts) instead. Moreover, they keep them fixed for all cases in the analysis, hampering the success rate of the neural network.

Secondly, the major achievement for application is the ability to classify insipient damage and its progression. The proposed method maximizes the Kappa coefficient in order to increase the 
success rate when the damage identification is being performed. As a consequence, the results obtained showed better than the previous approaches (De Oliveira \& Inman, 2016, 2017). The feasibility of the proposed method implies potential practical applications in composite materials where incipient damage exists. As a consequence, the method can lead to an increase in safety and reduce maintenance costs in SHM systems.

Despite the advantages, improvements of the proposed method still need to be investigated. The major problem of the current approach for application in a real-time solution is the time consumption. The PSO algorithm runs many particles, the time consumption is much longer than other FAN-based method presented in the SHM literature (De Oliveira \& Inman, 2016, 2017). To find the optimal parameters, PSO needs to run several iterations for each chosen particle which influences the total time. It is important to point out that we have only shown total time (training + testing), however if the training phase was conducted offline, the test phase may be successfully conducted on line. From a practical point of view, the majority of SHM applications run offline instead. However, fast learning has an important influence on applications on real-time SHM methods and this should be investigated in the future.

Another important limitation lies in the fact that the results present good performance for damage located within nearly $120 \mathrm{~mm}$ of the PZTs, a fault shared by many approaches in the field of SHM. This is especially true for composites because of their inherent damping. It is beyond the scope of this paper to discuss this limitation in any detail. Notwithstanding, further details about such limitations can be read in the follow references (De Oliveira \& Inman, 2016, 2017; Na \& Lee, 2012; Park et al., 2003). A key to overcome this limitation consists of adding extras PZTs to the structure in order to ensure that the maximum distances among them are of $120 \mathrm{~mm}$ (sensors network).

\section{Conclusion and prospects}

This paper has presented an exploration of the suitability of Kappa-PSO-FAN method applied to monitoring the progression of structural damage in composite structures. To date, there is no evidence of any another method, focusing in the same issues as presented here, which aims to monitor the damage progression based on the EMI technique along with Kappa-PSO-FAN method. In addition to the fact that the SHM-FAN based method is proposed here for the first time, the major contribution of this approach consists of a reliable method to selecting optimal FAN parameters by using the Kappa-PSO algorithm.

As a result, the optimum parameters significantly improved the success rate while the damage identification was being performed. As observed from these results, the proposed method was also successfully able to identify various structural conditions with higher accuracy rate when using the Kappa-PSO based method. It is important to highlight that each structural condition was identified with overall success rate of at least $75 \%$ which is considered an acceptable performance within the neural networks literature.

Comparing all methods, it can be clearly observed that the proposed method based on Kappa-PSO-FAN significantly enhances the overall success rate used to identify the progression of structural damage in composite structures. The intention of the paper is detecting incipient damage and its progression in composite materials and we believe that this objective was successfully accomplished within the limitation of distances from PZTs patches to damage of $120 \mathrm{~mm}$. Despite challenging, studies focusing on monitoring incipient damage constitute a cutting-edge issue in SHM systems which require continues thorough investigation.

Future work will be done through additional features other than the success rate for measuring the classifier efficiency. In addition, the full analysis of the error statuses that were presented during the supervised learning of fuzzy ARTMAP neural using the KappaPSO algorithm can lead to a more intelligent and efficient identification of the progression of structural damage, including damage classification.

Furthermore, an attribute selection technique based on the Kappa coefficient and the ARTMAP Fuzzy neural network, to extract the most significant attributes of the training base and generate an optimal subset of attributes, can lead to a significant improvement in the damage recognition module. It may also result in reducing the required time.

In addition an investigation of the variations of the PSO setup parameters (number of iterations, number of population particles, initial inertial weight, random parameters, cognitive and social parameters) needs to be addressed in order to improve the results and reduce the required time.

It is also recommended that further research will be undertaken in trying parallel computing techniques to optimize the required time consumption.

\section{Funding}

The authors would like to thank the CNPq, Brazilian Research Agency (Grant 248665/2013-8), and the University of Michigan through the Kelly Johnson Collegiate Chair fund.

\section{Acknowledgment}

The authors are grateful to the reviewer's valuable comments that improved the manuscript.

\section{References}

Ali, J. B., Saidi, L., Mouelhi, A., Morello, B. C., \& Fnaiech, F. (2015). Linear features election and classification using PNN and SFAM neural networks for a nearly on line diagnosis of bearing naturally progressing degradations. Engineering Applications of Artificial Intelligence, 42, 67-81.

Annamdas, V. G. M., \& Soh, C. K. (2010). Application of electromechanical impedance technique for engineering structures: Review and future issues. Journal of Intelligent Material Systems and Structures, 21(229), 41-59.

Araújo, N., de Oliveira, R., Ferreira, E. T., Nascimento, V., Akira, A. S., \& Bhargava, B. (2013). Kappa-Fuzzy ARTMAP: A feature selection based methodology to intrusion detection in computer networks. In Proceeding of 12th IEEE international conference on trust, security and privacy in computing and communications, 16-18 July, Melbourne, Australia (pp. 1-6).

Baptista, F. G., \& Vieira Filho, J. (2009). A new impedance measurement system for PZT-based structural health monitoring. IEEE Transactions on Instrumentation and Measurement, 58(10), 3602-3608.

Blanloeuil, P., Nurhazli, N. A. E., \& Veidt, M. (2016). Particle swarm optimization for optimal sensor placement in ultrasonic SHM systems. In Proceeding of SPIE 9804 nondestructive characterization and monitoring of advanced materials, aerospace, and civil infrastructure: Vol. 9804 (pp. 1-11). Las Vegas, Nevada, United States.

Brand, C., \& Boller, C. (1999). Identification of cycle cost reduction in structures with self-diagnostic devices. In Proceeding of the symposium on design for low cost operation and support: Vol. 37 (pp. 1-8). Ottawa, CA.

Carpenter, G. A., Grossberg, S., Markuzon, N., Reynold, J. H., \& Rosen, D. B. (1992). Fuzzy ARTMAP: A neural network for incremental supervised learning of analog multidimensional maps. IEEE Transactions on Neural Network, 3(5), 689-713.

Carpenter, G. A., Grossberg, S., \& Rosen, D. B. (1991). Fuzzy ART: Fast stable learning and categorization of analog patterns by an adaptive resonance system. Neural Networks, 4(6), 759-771.

Chaudhry, Z., Lalande, F., Ganino, A., Rogers, C. A., \& Chung, J. (1996). Monitoring the integrity of composite patch structural repair via piezoelectric actuators/sensors. In Proceeding of 36th AIAA/ASME, New Orleans, April 10-13, USA (pp. 2243-2248).

Cohen, J. (1960). A coefficient of agreement for nominal scales. Educational and Psychological Measurement, 20(1), 37-46.

Cortez, N. E., Vieira Filho, J., \& Baptista, F. G. (2012). A new microcontrolled structural health monitoring system based on the electromechanical impedance principle. Structural Health Monitoring, 1(2), 1-9.

De Oliveira, M. A., \& Inman, D. J. (2016). Simplified fuzzy ARTMAP network-based method for assessment of structural damage applied to composite structures. Journal of Composite Materials, 50(25), 3501-3514.

De Oliveira, M. A., \& Inman, D. J. (2017). Performance analysis of simplified fuzzy ARTMAP and probabilistic neural networks for identifying structural damage growth. Applied Soft Computing, 52, 53-63. 
De Oliveira, M. A., Vieira Filho, J., Lopes, V., Jr, \& Inman, D. J. (2016). A new approach for structural damage detection exploring the singular spectrum analysis. Journal of Intelligent Material Systems and Structures First published date: September-05-2016. doi:10.1177/1045389X16667549.

Duan, S. M., \& Ye, T. Q. (2002). Three dimensional frictional dynamic contact analyses for predicting low-velocity damage in composites laminates. Advances Engineering Software, 36(1), 9-15.

Eberhart, R. C., \& Shi, Y. (1998). Comparison between genetic algorithms and particle swarm optimization. In V. W. Porto, N. Saravanan, D. Waagen, \& A. E. Eiben (Eds.), Evolutionary programming VII. EP 1998. Lecture notes in computer science: Vol. 1447. Berlin, Heidelberg: Springer.

Garcia-Breijo, E., Garrigues, J., Sanchez, L. G., \& Laguarda-Miro, N. (2013). An embedded simplified fuzzy ARTMAP implemented on a microcontroller for food classification. Sensors, 13, 10418-10429.

Giurgiutiu, V., \& Zagrai, A. N. (2002). Embedded self-sensing piezoelectric active sensors for online structural identification. Journal of Vibration and Acoustics, $124,116-125$.

Granger, E., Renniges, P., Sabourin, R., \& Oliveira, L. S. (2007). Supervised learning of fuzzy ARTMAP neural networks through particle swarm optimization. Journal of Pattern Recognition Research, 1, 27-60.

Han, W., Xu, J., Wang, P., \& Tian, G. (2014). Defect profile estimation from magnetic flux leakage signal via efficient managing particle swarm optimization. Sensors, 14(6), 10361-10380.

Kasuba, T. (1993). Simplified fuzzy ARTMAP. AI Expert, 8(11), 18-25.

Kennedy, J., \& Eberhart, R. (1995). Particle swarm intelligence. In Proceeding of IEEE international conference on neural networks, 27 November-1 December, Perth, Western Australia (pp. 1942-1948).

Lewis, C. (2012). Commercial fleet forecast. In Global: 2012-2031 (pp. 1-8). FligthGlobal: London.

Liang, C., Sun, F. P., \& Rogers, C. A. (2014). Coupled electromechanical analysis of adaptive material systems - determination of the actuator power consumption and system energy transfer. Journal of Intelligent Material Systems and Structures, 5(1), 12-20.

Lima, F. P. A., Souza, A. S., Chavarette, F. R., Lopes, M. L. M., Turra, A. E., \& Lopes, V., Jr (2014a). Monitoring and fault identification in aeronautical structures using an ARTMAP-fuzzy-wavelet artificial neural network. Advanced Materials Research, 1025-1026, 1107-1112.

Lima, F. P. A. Souza, A. S. Chavarette, F. R. Lopes, M. L. M. Turra, A. E. \& Lopes, V., Jr (2014b). Analysis of structural integrity of a building using an artificial neural network ARTMAP-fuzzy-wavelet. Advanced Materials Research, 1025-1026, 1113-1118.

Lopes Jr, V., Park, G., Cudney, H. H., \& Inman, D. J. (2000). Impedance-based structural healthy with artificial neural networks. Journal of Intelligent Material Systems and Structures, 11(3), 206-214.

Na, S. \& Lee, H. K. (2012). Resonant frequency range utilized electro-mechanical impedance method for damage detection performance enhancement on composite structures. Composite Structures, 94(8), 2383-2389.
Na, S., \& Lee, H. K. (2013). Neural network approach for damage area location prediction of a composite plate using electromechanical impedance technique. Composites Science and Technology, 88, 62-68.

Palomino, L. V., Steffen, V., Jr, \& Finzi Neto, R. M. (2014). Probabilistic neural network and fuzzy cluster analysis methods applied to impedance-based SHM for damage classification. Shock and Vibration, 2014, 1-12.

Papatheou, E., Dervilis, N., Maguire, A. E., Antoniadou, I., \& Worden, K. (2015). A performance monitoring approach for the novel lillgrund offshore wind farm. IEEE Transactions on Industrial Electronics, 62(10), 6636-6644.

Park, G., Cudney, H., \& Inman, D. J. (2000). An integrated health monitoring technique using structural impedance sensors. Journal of Intelligent Material Systems and Structures, 11(6), 448-455.

Park, G., \& Inman, D. J. (2007). Structural health monitoring using piezoelectric impedance measurements. Philosophical Transactions of Royal Society, 355(1851), 373-392.

Park, G., Sohn, H., Farrar, C., \& Inman, D. J. (2003). Overview of piezoelectric impedance-based health monitoring and path forward. Journal of Shock and Vibration Digest, 35(6), 451-463.

Qian, X., Cao, M., Su, Z., \& Chen, J. (2012). Hybrid particle swarm optimization (PSO)-simplex algorithm for damage identification of delaminated beams. Mathematical Problems in Engineering, 2012, 1-12.

Santos, A., Silva, M., Santos, R., Figueiredo, E., Sales, C., \& Costa, J. C. W. A. (2016). A global expectation-maximization based on memetic swarm optimization for structural damage detection. Structural Health Monitoring, 15(5), 610-625.

Saxena, A., \& Saad, A. (2007). Evolving an artificial neural network classifier for condition monitoring of rotating mechanical systems. Applied Soft Computing, 7(1), 41-454.

Selva, P., Cherrier, O., Bundinger, V., Lachaud, F., \& Morlierb, J. (2013). Smart monitoring of aeronautical composites plates based on electromechanical impedance measurements and artificial neural networks. Engineering Structures, $56,441-454$.

Specht, D. F. (1998). Probabilistic neural networks for classification, mapping, or associative memory. Neural Networks, $1,525-532$.

Su, H., Wen, Z., Chen, Z., \& Tian, S. (2016). Dam safety prediction model considering chaotic characteristics in prototype monitoring data series. Structural Health Monitoring, 15(6), 639-649.

Sun, F. P., Chaudhry, Z., Liang, C., \& Rogers, C. A. (1995). Truss structure integrity identification using PZT sensor-actuator. Journal of Intelligent Material Systems and Structures, 6, 134-139.

Vakil-Baghmisheh, M-T., \& Pavesic, N. (2003). A fast simplified fuzzy ARTMAP network. Neural Processing Letters, 17(3), 273-316.

Vieira Filho, J., Baptista, F. G., \& Inman, D. J. (2011). Time-domain analysis of piezoelectric impedance-based structural health monitoring using multilevel wavelet decomposition. Mechanical Systems and Signal Processing, 25, 1550-1558.

Yang, Y., Liu, H., Annamdas, V. G. M., \& Soh, C. K. (2009). Monitoring damage propagation using PZT impedance transducers. Smart Materials and Structures, 18, 1-9.

Zagrai, A. N., \& Giurgiutiu, V. (2001). Electromechanical impedance method for crack detection in thin plates. Journal of Intelligent Material Systems and Structures, 12 709-718. 